\title{
Review and Future Prediction on the Models of Mortality Rate of Covid-19
}

\begin{abstract}
Yalong Zhang
University of Washington

*Corresponding author. Email: yalonz@uw.edu

ABSTRACT

Before the vaccine for Covid-19 was invented, lots of studies and models are down to determine about what specific factors could influence the mortality rate of Covid-19. However, after vaccination process have been pushed to the majority of the population, it is necessary to reconsider these models for mortality and add vaccination as a main factor. Through this review, the author will firstly summarize the exist main factors that could affect the mortality rate: Age, gender, race. Then take vaccine into account and review the reason why vaccination should be considered as a main factor based on the existing factors. Then the author gives suggestions about where and how to focus on future studies of mortality rate of Covid-19. Future Studies needs to focus more on how vaccination rate correlates with other factors that influences Covid-19's death rate.
\end{abstract}

Keywords: Mortality rate, Covid-19, prediction, main factors.

\section{INTRODUCTION}

The first reported case of the novel coronavirus-2019 disease could be traced in November 2019. Since then, it has spread to all over the world, and caused hundreds of thousands of deaths in both North America and Europe. There are in total 257,832,881 cases and 5,168,069 deaths in worldwide range [1]. The case fatality rate is about $2 \%$. Ever since the covid-19 became a global pandemic, it is urge for scientists to find out what would be the fatality rate of this disease so that the public can treat covid-19 in a more clear and scientific way. Many researchers focused on analyzing factors such as age and gender, and then construct model to predict the death rate. However, many of them did not consider about the vaccination rate. It has already been almost one year since the first covid-19 vaccine got approved [2]. Take Pfizer as an example: it is an encapsulated mRNA Vaccine. Its mRNA Encoding for the Spike protein is protected in a lipid nanoparticle. Once absorbed, the cell expresses the Spike protein resulting in an immune response. Pfizer Vaccine is reported to be $95.3 \%$ effective in preventing severe disease as defined by the U.S. Food and Drug Administration [3]. Then it is important to take vaccination rate of the population into consideration since it could prevent severe symptoms so that death rate can be affected.

\section{METHOD}

There are dozens of essays that stands out from the data base. A statistical analysis is conducted to review the association of Sex, Age, and Comorbidity [4]. We focused on the part where age is particularly mentioned and examined to help review the relationship between age and mortality of Covid-19. Then it is possible to gather information that we need to make predictions and suggestions on how vaccine plays a role among them. In addition, a study focuses on the putative role of sex hormones and $\mathrm{X}$ chromosome to Covid-19 [8]. This study reveals the fact that men are more susceptible to Covid-19 than female, according to the data from Italy. Then it hypothesizes that it is related to the differences of hormone and $\mathrm{X}$ chromosome between male and female. Using this hypothesis, we are able to deduce a hypothesis for the effectiveness of vaccine on male and female and suggest directions for future studies. What is more, in those essays, some of them focused on the racial factors' influence on Covid-19 mortality. One of the essays did a single-center retrospective cohort study of Covid-19 patients hospitalized in a NYC hospital within a 2-month period. This study reflected on the mortality rate based on different race to investigate how race affects the death rates. People evaluated this essay to understand what the cause of this differences between races might be and then correlated with other models and experiment to predict 
on how vaccine react to different race people. Finally, the dashboard for Covid-19 of King County in Washington State gives the statistics of the vaccinated populations. By gathering and calculating the death rate of both vaccinated and unvaccinated people, we are able to make assumptions about the effectiveness of vaccine on preventing severe symptoms or death of Covid-19 patients. In addition to that, the dashboard also gives suggestions for getting the vaccine. Together, we can make suggestions for future studies.

\section{FINDINGS}

\subsection{Age Factor}

Studies has shown that age is a main factor for fatality rate in the Covid-19 pandemic. For male patients, age $>50$ years, were significantly associated with increased risks of mortality [4]. A study in New York city of 393 Covid-19 infection cases had shown that the median age of these patients is 62.2 [5]. 40 of these patients had died and the fatality rate is $10.2 \%$, with 93 people in the study have incomplete outcome data. In this case, it is clearly that the mortality among elder populations who are infected with Covid-19 is way higher the general mortality of about $2 \%$ [1]. Reason behind that is considered to be that elder people have weakened immune system. Elder population have higher rate of comorbidity [6]. It also uncovered that elder people have faster period to develop severe symptoms. People that are 70 years or old have a median day of 11.5 days from the first symptom to death, compared to the median day of 20 days of people under 70 years old [7]. By the Covid-19 Dashboard of King County in Washington, $85 \%$ of the deaths due to Covid- 19 have the age great or equal to 60 . For specifically, $16 \%$ of the deaths are within age $60-69$ years; 235 of the deaths are within age $70-79$ years; and $46 \%$ of the deaths are within age over 80 .

\subsection{Gender Factor}

Following by the different mortality rate with shifting aging, gender is also a main factor related to death rate. In an analysis from December 2020 on 238,709 patients of Covid-19 in Italy, the mortality rate is $17.7 \%$ in men and $10.8 \%$ in women, with $59 \%$ of total death being men [8]. This analysis suggesting that men generally have higher risk to develop severe symptoms of Covid-19 than women. In addition, men have higher mortality from Covid-19 than women in almost all aging groups in Europe. This sex differences increased from age $<60$ years to 60-69 years and decreased after that [9]. This phenomenon might be due to the different role of sex hormones and $\mathrm{X}$ chromones between men and women. Low levels of certain kinds of androgens enables women to keep and at low levels TMPRSS2 expression, which is one of the actors of Covid-19 Infection. This factor, thus, creates a natural protection for the further development of Covid-19 infection [8]. What is more, sex differences include more factors that could affect the mortality of Covid-19. For example, men have higher rate of smoking than women, which can lead to lung issues that could cause severe symptoms when being infected with Coronavirus.

\subsection{Race Factor}

According to a study that analyzed the dataset of 50 countries with difference race distributions and the corresponding Covid-19 infection and fatality rate, in countries that are $1 \mathrm{SD}$ above the Black-White segregation mean, the Black death rate is $8 \%$ higher than White death rate of Covid-19 [10]. In the overall study, Blacks and Hispanics have the highest mortality rates. While socioeconomic factors might influence the study, another study of in-hospitality mortality rate concludes that Hispanics have the highest rate of experiencing inhospital mortality [11]. On the contrary, in the Covid-19 summary dashboard of King County in Washington State, there are 19920 cases of Asians, 16415 cases of Blacks, 30760 cases of Hispanics, and 69162 cases of Whites. At the same time, the deaths numbers are 292 deaths of Asians, 172 deaths of Blacks, 173 deaths of Hispanics, and 1300 cases of Whites [12]. then we have Whites have the highest mortality rate of $1.9 \%$, with Hispanics has the least mortality rate of $0.6 \%$.

\subsection{Vaccination factor}

The actual effects of vaccination are still under investigation. Some studies point out that due the uncertainty of vaccines, there is a huge challenging for modeling include vaccination as a factor [13]. By adjusting people's susceptibility and probability to develop symptoms after infections, vaccination can be included in the model that reduce the probability of progressing to severe disease or death. By the Covid Vaccination Summary of King County conducted by the Public Health department, $72.9 \%$ of the total population in King County have completed their vaccination series [14]. Then the Public Health department of King County also deduced a study of the outcomes of Vaccination. For the total of 796 deaths, 673 are not fully vaccinated and 123 are fully vaccinated. They concluded that since $1 / 17 / 2021$, people who are not fully vaccinated are 11.9 time more likely to die due to Covid-19 related illness than people who are fully vaccinated [15]. Among people not fully vaccinated in King County, there have been 0.37 new deaths per 100,000 residents per day, compared to 0.03 new deaths per 100,000 residents per day among fully vaccinated individuals. In addition, they noted that deaths among unvaccinated have tended to affect younger and healthier people than the comparatively rare deaths among vaccinated people. 


\section{DISCUSSION}

Firstly, age is correlated to comorbidity when being considered as a factor to cause mortality in Covid-19 cases. Then it is important to determine the effects of different vaccines on the comorbidity. A study that used the vaccinated population as both the experiment and control groups show that the vaccine (Pfizer) the overall great effectiveness over population of age 16-18 years [16]. And for elder people (above 80), they observe slightly lower effectiveness. In addition, it shows that people with certain chronic comorbidity like type 2 diabetes and high blood pressure would have low effectiveness on the vaccine. However, they still conclude that the vaccine is still effective even for people with those negatively associated factors. The vaccine has a $90 \%$ effectiveness to prevent infected populations to develop severe disease or death. Then we summarize that vaccine is a key point when considering age as a factor to build a model to predict pandemic spread, since vaccine shows a general effectiveness for people with all age.

There are few studies to show that the vaccine has different effects on Covid-19 mortality rate between different sexualities. The mean concentration of $\mathrm{IgG}$ geometric concentration mean after vaccination is 64.6 $\mathrm{AU} / \mathrm{ml}$ in men and $75.9 \mathrm{AU} / \mathrm{ml}$ in women, showing that the vaccine has slightly higher effectiveness in women. On the other hand, vaccine do show a different effect on the infection effectiveness between men and women. Men appears to have a $33 \%$ reduction in developing Covid-19 compared to women [17]. But this is far from concluding that mortality rate is affected at the same circumstance as infection rate. In addition, social difference must be taken into consideration since men and women have different inclinations on whether to get the vaccination. In this situation, a certain experiment group and a control group of vaccinated population can be biased. Future studies should aim on finding the implicit pharmacological relationship of vaccine and gender. Like the study about the hormones and Covid-19 [9], similar study can be established to determine whether gender plays a role in influencing the effectiveness of Covid-19 Vaccines.

Through the findings of race factors, we see a controversy between the study of in-hospital mortality rate and the Covid-19 summary dashboard of King County. The in-hospital case study reflects that Hispanic have the highest mortality rate and the dashboard of King County shows the opposite. The reason behind that might be because of the different distribution of the hospitalization rate of different races. It is reasonable to deduce that with high mortality rate for Hispanics in hospital is because these Hispanic patients who were sent to hospitalizations had already developed severe diseases that could lead to death. Expanding to the whole population of Hispanics who are infected with Covid-19, we just had mild symptoms. Then for these groups, hospitalization is not needed, and thus mortality of Covid-19 is comparably low. For future studies, since there is no clear evidence that race is a clear factor to influence vaccine, not even clear to affect the Covid-19, a more general study consisting of all the populations of infected people for a certain race is needed to give conclusion about how race being a factor in vaccine effectiveness.

For the study that generated by the public health department which suggests that Vaccination help reduce the mortality rate by 11.9 time, it didn't include any specific methodology and models to illustrate how it draws this conclusion. It simply calculated the rate for the mortality number which could be totally biased. For example, the groups that are not fully vaccinated might be the group with other comorbidity who are not suggested to get the vaccine. And this group is more likely to develop severe diseases.

\section{CONCLUSION}

Due to the fact that vaccines have just come out for months, and medical studies aren't sufficient to show the exact relationship between vaccines and the above factors. On the other hand, exist studies of Covid-19 and the group studies of fully vaccinated population give us some suggestions and directions of future studies. For gender factor, examination of different hormones and the $\mathrm{X}$ chromone have influence on the vaccine effectiveness. For age factor, current group studies have shown that vaccines have great effectiveness on preventing mortality. However, future studies need to focus on the effectiveness of vaccine especially for elder groups like people with age over 80 years. Since race factor has a lot to do with socioeconomic factor, it is hard to come out with a model considering all possible variables. If possible, pharmacological study can be established to examine different result of vaccine on the mortality rate of different races. Finally, it is important to show that different kinds of vaccines may have different effectiveness on preventing death of Covid-19. In the US, the main vaccine kind is the mRNA kind, which has the most effectiveness. Other vaccines, like Sinovac, is the kind that is called the Live attenuated vaccine, which is constructed by inactive virus. Different types of vaccines may have totally different effects on the mortality rate of Covid-19. Thus, future studies should carefully state about this factor.

\section{REFERENCES}

[1] covid live update data, 2020. Doi: https://www.worldometers.info/coronavirus/

[2] A Timeline of COVID-19 Vaccine Developments in 2021. 2021. https://www.ajmc.com/view/a- 
timeline-of-covid-19-vaccine-developments-in2021

[3] Pfizer and BioNTech Confirm High Efficacy and No Serious Safety Concerns Through Up to Six Months Following Second Dose in Updated Topline Analysis of Landmark COVID-19 Vaccine Study; 2021.

https://www.businesswire.com/news/home/202104 01005365/en/

[4] Biswas M. Association of Sex, Age, and Comorbidities with Mortality in COVID-19 Patients: A Systematic Review and Meta-Analysis. 2020. https://doi.org/10.1159/000512592

[5] Parag Goyal. Clinical Characteristics of Covid-19 in New York $\quad$ City. 2020. https://www.nejm.org/doi/10.1056/NEJMc201041 9

[6] Paola Senia. Survey on COVID-19-related mortality associated with occupational infection during the first phase of the pandemic: A systematic review. $2021 . \quad$ https://www.spandidospublications.com/10.3892/etm.2021.10932

[7] Weier Wang. Updated understanding of the outbreak of 2019 novel coronavirus (2019-nCoV) in Wuhan, China.

2020. https://onlinelibrary.wiley.com/doi/full/10.1002/jm v. 25689

[8] C. Foresta. Gender susceptibility to COVID-19: a review of the putative role of sex hormones and $\mathrm{X}$ chromosome. (2021):951-956: https://link.springer.com/article/10.1007/s40618020-01383-6

[9] Linda Juel Ahrenfeldt. Sex and age differences in COVID-19 mortality in Europe. 2021 393-398: https://link.springer.com/article/10.1007/s00508020-01793-9

[10] Douglas S. Massey. Using machine learning to estimate the effect of racial segregation on COVID19 mortality in the United States; .2020. 118 (7): https://www.pnas.org/content/118/7/e2015577118. full

[11] Benjamin D. Renelus. Racial Disparities in COVID19 Hospitalization and In-hospital Mortality at the Height of the New York City Pandemic. 2021. 1161-1167:

https://link.springer.com/article/10.1007/s40615020-00872-x

[12] Daily COVID-19 outbreak summary dashboard. 2021. https://kingcounty.gov/depts/health/covid19/data/daily-
summary.aspx\#collapse4A25CFF959E04B63BEA FD5045EDCBD05

[13] Cliff C. Kerr. Covasim: An agent-based model of COVID-19 dynamics and interventions; (2021): https://journals.plos.org/ploscompbiol/article?id=1 0.1371/journal.pcbi.1009149

[14] Covid-19 Vaccination Summary for King County. 2021.https://tableaupub.kingcounty.gov/t/Public/vi ews/COVIDVaccinePublicDashboardV2/FrontPag e?\%3AshowAppBanner=false \&\%3Adisplay_count $=$ n\&\%3AshowVizHome $=$ n $\& \% 3$ Aorigin $=$ viz_share _link\&\%3AisGuestRedirectFromVizportal=y\&\%3 Aembed $=y$

[15] Covid-19 Outcomes by Vaccination Status. 2021. https://public.tableau.com/views/COVID_Vaccinat ion_Status/VaxBrk?:embed=y\&:toolbar=n\&:displa y_count=n\&:showShareOptions=n\&:showVizHom $\mathrm{e}=\mathrm{n}$

[16] Idan Yelin. Associations of the BNT162b2 COVID19 vaccine effectiveness with patient age and comorbidities. 2021. https://www.medrxiv.org/content/10.1101/2021.03 $.16 .21253686 \mathrm{v} 2$.full-text

[17] Alessia Bignucolo. Sex Disparities in Efficacy in COVID-19 Vaccines: A Systematic Review and Meta-Analysis. 2021. https://www.mdpi.com/2076393X/9/8/825/htm 